\title{
Persistent Tracheostomy after Organ Preservation Protocol in Patients Treated for Larynx and Hypopharynx Cancer
}

\author{
Carlos Miguel Chiesa Estomba ${ }^{1}$ Frank Alberto Betances Reinoso ${ }^{1}$ Virginia Martinez Villasmil ${ }^{1}$ \\ Maria Jesus González Cortés ${ }^{1}$ Carmelo Santidrian Hidalgo ${ }^{1}$
}

1 Otorhinolaryngology - Head and Neck Surgery Department,
Complexo Hospitalario Universitario de Vigo, Vigo, Pontevedra,
Spain

Int Arch Otorhinolaryngol 2017;21:377-381.

\begin{abstract}
Address for correspondence Carlos Miguel Chiesa Estomba, Consultant, MD, MSc, Department of Otorhinolaryngology - Head and Neck Surgery, Complexo Hospitalario Universitario de Vigo, rua Doña Cristina, \#2, 2do H. 36214, Vigo, España

(e-mail: chiesaestomba86@gmail.com).
\end{abstract}

\begin{abstract}
Keywords

- larynx

- cancer

- radiotherapy

- chemotherapy

- tracheostomy

Introduction Squamous cell carcinoma of the larynx is currently the second most common malignancy of the airway after lung cancer, and hypopharyngeal cancer accounts for fewer than $5 \%$ of head and neck cancers. The nonsurgical options for patients with this disease are related to significant long-term toxicities and the need for persistent tracheostomy, which adversely affects the quality of life of these patients. Objective To evaluate the need for tracheostomy, and the influence of this in the overall and specific survival rates of patients diagnosed with all stages of laryngeal carcinoma treated by chemoradiotherapy.

Methods A retrospective study of patients diagnosed with laryngeal carcinoma was performed according to the criteria of the Union for International Cancer Control (UICC) and the American Joint Committee on Cancer (AJCC) 7th edition, in a tertiary hospital.

Results A total of 21 patients were evaluated, 8 patients required a tracheotomy (31\%) during the treatment protocol, 7 (35\%) men and 1 (100\%) women. According to subsite $4 / 4$ patient with glottis cancer $(\mathrm{p} \leq 0.001), 2 / 10$ patients with supra glottis cancer and 2/7 patients with hypopharyngeal cancer. During follow up, just in 1 patient was possible to close the tracheostomy.

Conclusion Persistent tracheostomy dependence after primary chemoradiation increases significantly the morbidity, and decreases the quality of life of those patients. Patients with glottis cancer are prone to need a tracheostomy, but no statistical difference regarding the oncological stage and the need for a tracheostomy were detected. A more thorough selection of the patients is needed to improve the quality of life and reduce permanent tracheostomy dependence.
\end{abstract}

\section{Introduction}

Squamous cell carcinoma of the larynx is currently the second most common malignancy of the airway after lung cancer, ${ }^{1}$ and hypopharyngeal cancer accounts for fewer than
$5 \%$ of head and neck cancers. ${ }^{2}$ Different treatment options exist, and they can be surgical or nonsurgical, such as radiotherapy (RT) or chemoradiotherapy (CRT).

In advanced stages of the disease, the treatment strategy has evolved to combine functional preservation (speech, received

November 20, 2016 accepted after revision

February 5, 2017

published online

April 3, 2017
DOI https://doi.org/

10.1055/s-0037-1601416. ISSN $1809-9777$.
Copyright $(2017$ by Thieme Revinter

Publicações Ltda, Rio de Janeiro, Brazil
License terms

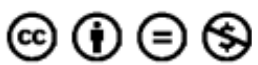


swallowing, and respiration) and loco-regional control to increase survival rates. Despite this, laryngeal cancer remains today as the only tumor for which life expectancy has declined in recent decades, and this is thought to be due to the organ preservation protocols. ${ }^{3,4}$ The prognosis of hypopharyngeal cancer continues to be worse than that of other types of cancer due to its late presentation, with $\sim 70$ $90 \%$ of patients having stage III or IV diseases at the time of presentation, and this is at least in part attributable to the anatomy, the rapid lymphatic spread of these tumors and the location of the hypopharynx. ${ }^{5}$

Although chemoradiation protocols can achieve loco-regional control, ${ }^{6,7}$ many of the patients experience significant long-term toxicities, ${ }^{8,9}$ and their quality of life is adversely affected. Speech and swallowing dysfunctions are the most commonly reported complications after the organ preservation protocols. However, the need for persistent tracheostomy also represents a treatment complication, due to the financial, speech, and swallowing implications, which adversely affect the quality of life of these patients. ${ }^{10}$

For this reason, the aim of this study is to retrospectively evaluate the rate of persistent tracheostomy in a series of patients treated by CRT for all stages of laryngeal carcinomas in a tertiary university hospital.

\section{Material and Methods}

A retrospective analysis of those patients diagnosed with a laryngeal carcinoma (T1, T2, T3, T4), $\mathrm{N}-/+, \mathrm{M}-/+$, was performed according to the criteria of the Union for International Cancer Control (UICC) and the American Joint Committee on Cancer (AJCC) 7th edition, in a tertiary hospital. Patients treated with radiotherapy or radiotherapy + chemotherapy between January 2009 and March 2012 were included. The identification of the cases was achieved by a search in the medical records of our department using the codes from the International Classification of Diseases (ICD)10. No approval from the ethics committee of our institution was needed, due the retrospective nature of our study.

Demographic data (age, gender), medical history, oncological stage, information on diagnostic tests, histological findings and complications were obtained through a review of the medical records.

Radiation therapy was performed according to the protocols established in our hospitals with intensity modulated radiation therapy (IMRT). The dose used was 70 to 72 Gray (Gy) over the primary site and clinically positive neck disease, and 50 Gy for clinically negative cervical lymph node to approach the risk of regional metastasis. Chemotherapy regimens were platinum-based and delivered weekly during the radiation therapy to standard area-under-the-curve dosing.

Before the treatment, all cases were discussed by the members of an interdisciplinary committee of head and neck tumors. Patients with suspicion of malignant lesions were scheduled for laryngeal microsurgery with biopsy. After this, the best treatment option according to the extension of the disease and the comorbidities was proposed to the patient, and both clinician and patient decided on the best option to treat the disease.

In our service, patients treated for head and neck malignant tumors are followed-up for at least 5 years at the Department of Head and Neck Oncology. However, for this study, we considered a group of patients that had been followed-up for a minimum of 36 months.

Statistical analysis was performed using the Statistical Package for the Social Sciences software for Windows, version 20.0 (IBM SPSS, Chicago, Il, US). The quantitative variables in the study were expressed as mean \pm standard deviation (SD). The differences between the groups were studied using the chi-square test, while the correlations between the variables were analyzed using the Spearman's rank correlation coefficient. We calculated the overall survival and the specific survival rates using the Kaplan-Meier survival analysis. As reference for the overall survival, we used the survival time from the surgery until the last followup visit or date of death, regardless of cause. For the specific survival, we considered the time between the surgery until death caused by the tumor, or until a total laryngectomy was performed. We calculated the influence of various factors on survival using the log-rank method.

\section{Results}

Total 21 patients were evaluated, 20 (95.2\%) men and 1 woman $(4.8 \%)$. The average age was 60.9 years $( \pm 9.89=$ Min. 45/Max. 83). A total of 18 patients (85.7\%) consumed $>70 \mathrm{~g}$ of alcohol per day. All of them (100\%) were smokers who consumed more than 40 packs per year. Six patients (28.5\%) were diabetic, and 14 (66.7\%) hypertensive. The mean follow-up was 36 months $( \pm 29.2=$ Min: 6/Max: $36)$. Regarding the oncological stage, 14 patients (66.6\%) were on CT3, and 7 (33.3\%) were on CT4. A total of 8 patients (38.1\%) were classified as N0; $1(4.8 \%)$ as $\mathrm{N} 1 ; 10(47.61 \%)$ as $\mathrm{N} 2$; and $2(9.5 \%)$ as N3. There were $2(9.5 \%)$ cases of distant metastases.

Among all patients, 8 (31\%) required a tracheotomy during the treatment protocol, 7 (35\%) men and 1 (100\%) woman. Regarding the subsite, 4 out of 4 patients with glottis cancer ( $p \leq 0.001$ ), 2 out of 10 patients with supraglottis cancer, and 2 out of 7 patients with hypopharyngeal cancer required tracheostomies. The overall rate of tracheostomy before the primary organ preservation protocol was of $23.8 \%$ ( 5 patients) due to dyspnea before starting the treatment, and another 3 patients (14.2\%) received new tracheostomies for airway compromise in the first 12 months after the primary chemoradiation ( $p=0.162$ ) due to laryngeal edema and dyspnea. According to the oncological stage, 4 out of 14 patients with T3 stage diseases and 4 out of 7 patients with T4 stage diseases received a tracheostomy ( $p=0.648$ ) during all of the follow-up. Among the 5 patients who required tracheostomies before receiving chemoradiation, 2 had T3 stage tumors, and the tumoral subsite affected was the glottis for one patient and the supraglottis for the other; and 3 patients had T4 stage tumors, and the affected subsite was different for each one of them (glottis, 


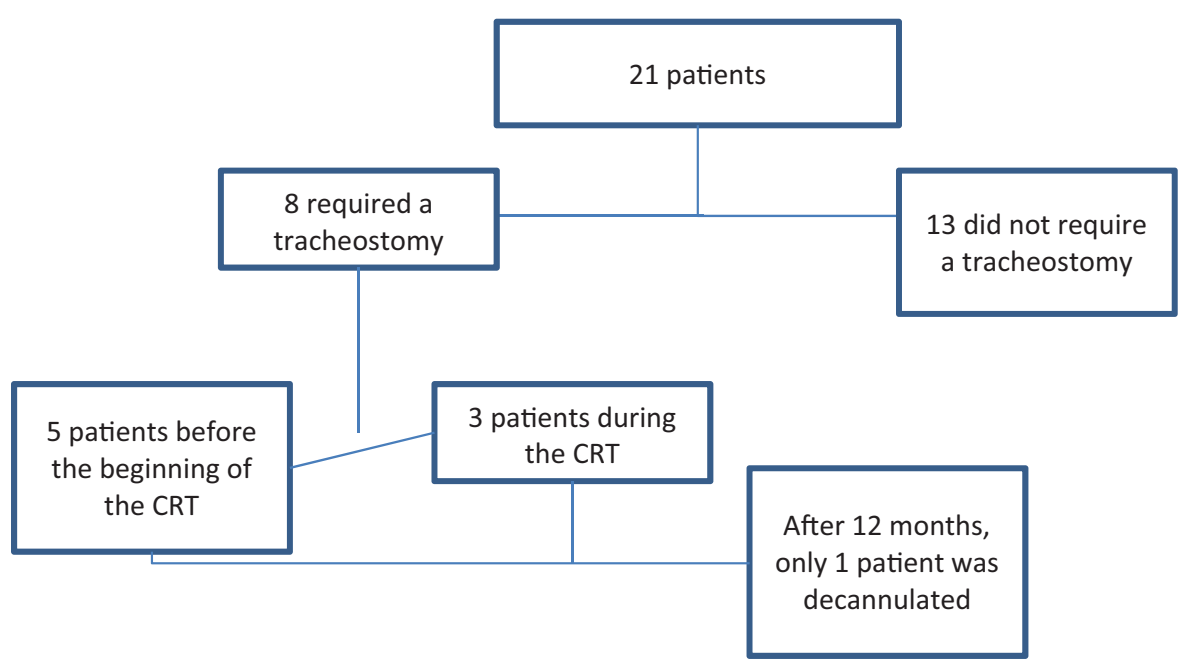

Fig. 1 Patients included in the study according the need for tracheostomy. Abbreviation: CRT, chemoradiotherapy.

supraglottis and hypopharynx) $(p=0.269)$. During the follow-up, we could only remove and close the tracheostomy of one patient (-Fig. 1). Data according to tumoral subsite, $\mathrm{T}$ stage and need for tracheostomy before or during the organ preservation protocol can be seen on - Table 1 .

Regarding survival, in the group of patients who needed a tracheotomy during the treatment, the 3-year overall survival rate was $25 \%$, while the 3 -year disease-specific survival rate was $12.5 \%$. In the group of patients who did not need a tracheostomy, the 3-year overall survival rate was $38.5 \%$, and the 3 -year disease-specific survival rate was $15.4 \%$. There were no statistically significant differences between both arms according to the overall $(p=0.551)$ or specific survival rates $(p=0.779)$ (-Figs. 2-3). During the follow-up, 3 patients (14.2\%) with tumors affecting the glottis needed a total laryngectomy performed to achieve local control. Out of these patients, two were in the no tracheostomy group, and one was in the no tracheostomy group.

\section{Discussion}

During the past 30 years, the therapeutic options for patients with laryngeal cancer have changed. However, the best approach is still a controversial issue, and many accepted surgical and non-surgical oncological treatments have been currently established.

In the past, the treatment of advanced tumors included surgery with or without radiotherapy according to nodal involvement and resection margins as the main therapeutic option. Nowadays, non-advanced carcinomas and selected advanced-stage tumors are managed with conservative surgery, such as open partial laryngectomy, transoral CO2 laser microsurgery (TLM), transoral robotic surgery (TORS), plus radiotherapy or chemoradiotherapy. ${ }^{11}$ However, in advanced tumors, if laryngeal preservation is the main goal, the patients are treated with concurrent CRT. ${ }^{12}$ Although this regimen has demonstrated efficacy in preserving the larynx, several of the patients submitted to it have ultimately required salvage laryngectomy ${ }^{13}$ or suffer from present long-term sequelae.

The Veterans Affairs Laryngeal Cancer Study Group ${ }^{14}$ findings published in 1991 heralded a shift in emphasis toward organ preservation via CRT for treatment of advanced laryngeal cancer. Moreover, the long-term results of the Radiation Therapy Oncology Group (RTOG) 91-11 trial show that concomitant CRT obtains better local control and a higher laryngeal preservation rate than radiotherapy alone or preceded by induction chemotherapy. ${ }^{15}$

Table 1 Data according to tumoral subsite, T stage and need for tracheostomy before the treatment or during the organ preservation protocol

\begin{tabular}{|l|l|l|l|l|l|}
\hline Tumoral Site & $\begin{array}{l}\text { Tumoral } \\
\text { stage }\end{array}$ & $\begin{array}{l}\text { Pre-treatment } \\
\text { tracheostomy }\end{array}$ & $\begin{array}{l}\text { Treatment } \\
\text { tracheostomy }\end{array}$ & $\begin{array}{l}\text { No } \\
\text { tracheostomy }\end{array}$ & $\begin{array}{l}\text { Total } \\
\text { subsite \% }\end{array}$ \\
\hline Supraglottis & T3 & 1 & 0 & 7 & 47.61 \\
& T4 & 1 & 0 & 1 & 19.04 \\
\hline Glottis & T3 & 1 & 1 & 0 & 0 \\
\hline Hypopharynx & T4 & 1 & 1 & 3 & 33.3 \\
& T3 & 0 & 0 & 2 & 13 \\
\hline
\end{tabular}




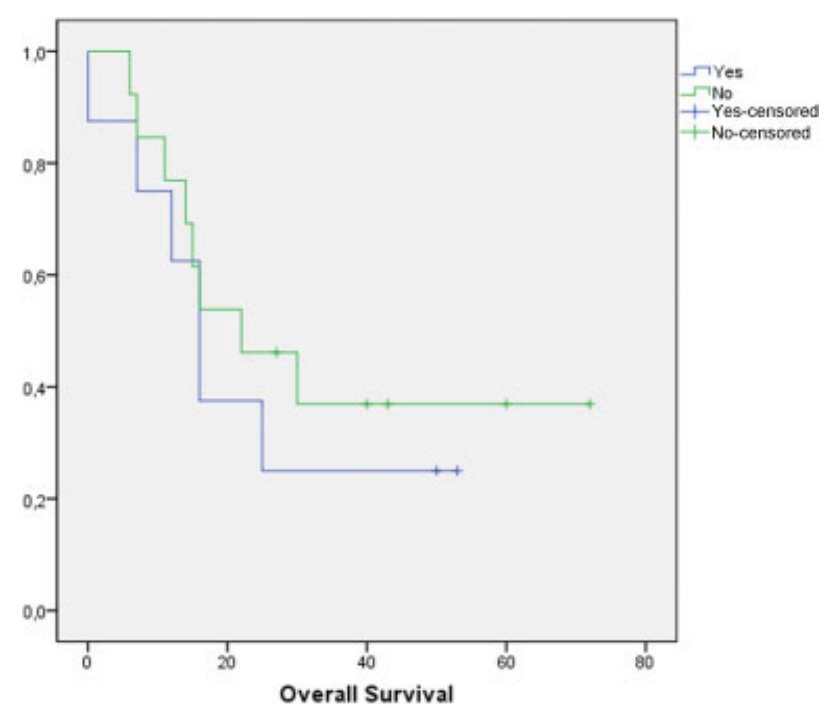

Fig. 2 Kaplan-Meier three-year overall survival according the need for tracheostomy.

However, the use of nonsurgical techniques as the primary therapy for advanced larynx cancer has been associated with reductions in survival. Hoffman et al reported a $3.4 \%$ drop in 5-year survival rates in 1994-1996 versus 19851990 in a National Cancer Database outcomes study. ${ }^{3}$

Despite this, the need for tracheostomy is acknowledged as an adverse prognostic factor. Larynx-preserving strategies are still being utilized in this population because of the confirmation of survival equivalence ${ }^{16}$ and the powerful desire of the patients to preserve their voices. ${ }^{17}$

In a study performed by Stanton et al, severe laryngopharyngeal dysfunction, defined as a persistent need for gastrostomy or tracheostomy, was present in $36 \%$ of the patients who underwent organ preservation treatments for advanced laryngeal malignancies and remained disease-free at 6 months after the completion of the therapy protocols. ${ }^{18}$ But, as we know, persistent tracheostomy requirement is also associated with poor functional outcome, requiring salvage surgery in many cases. ${ }^{13}$ Another study performed by Hutcheson et al studied the outcomes of patients who were rendered disease-free after an organ-sparing treatment but subsequently underwent elective total laryngectomy, and found that $39 \%$ of these individuals were tracheostomy-dependent before the removal of their larynges. ${ }^{19}$

In another study performed by Tennant et al, in patients with stage III or IV laryngeal or hypopharyngeal squamous cell carcinoma, $34 \%$ of them had tracheostomies performed on the, before the initiation of the treatment. The majority of the tracheostomies were performed for immediate or impending airway compromise. Moreover, they included patients who presented with $\mathrm{T} 1$ or T2 diseases, and they found that none of them were tracheostomy-dependent at the 12-month follow-up time interval. ${ }^{13}$

In our study, 5 (23.8\%) patients had tracheostomies performed before the initiation of the treatment, and another 3 (14.2\%) patients had tracheostomies performed during the

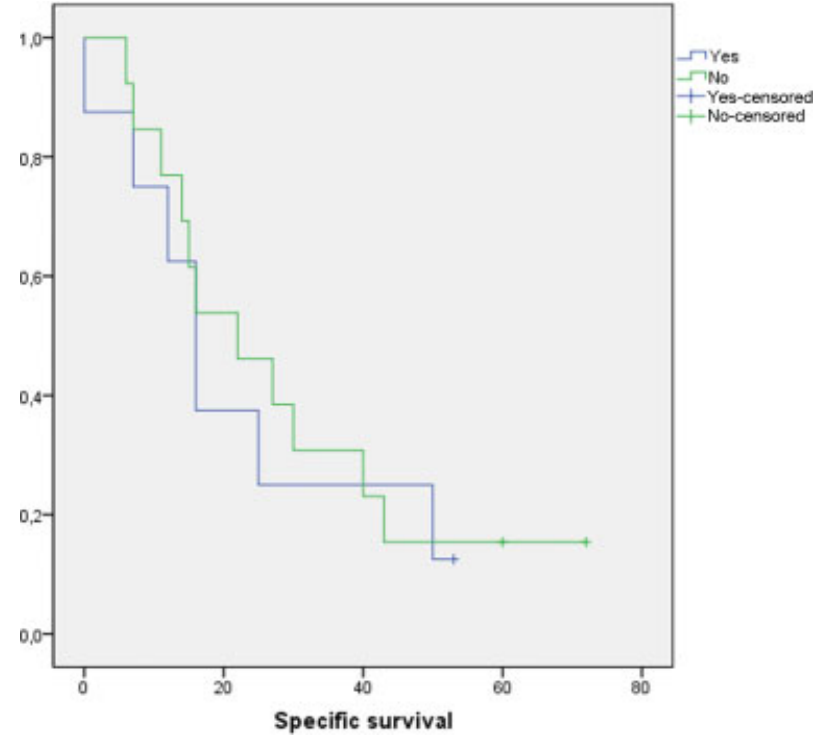

Fig. 3 Kaplan-Meier three-year specific survival according the need for tracheostomy.

treatment. After 12 months of treatment, 7 (33.3\%) patients were tracheostomy-dependent, a result similar to those reported in the literature, and, as we expected, patients with tumors involving the glottis were more likely to have a tracheostomy $(p \leq 0.001)$ ( - Fig. 1) performed on them. However, we did not find differences regarding tumoral stage, subsite and the need for tracheostomy $(p=0.648)$. Moreover, there were no statistically significant differences between both arms according to the overall $(p=0.748)$ and specific survival rates $(p=0.936)$.

However, our study has several limitations: primarily, it is a retrospective analysis, and the small sample size may limit the validity of our results. A short follow-up period (36 months) and the fact that no quality of life questionnaires were administered to the patients also limit our study. However, we only included those patients on advanced oncological stages, and a worse prognosis can be expected in more advanced tumors. Therefore, we suggest the need for prospective studies about the influence of permanent tracheostomy in these patients, because in a new era in which medical advances help to increase life expectancy, it is important to know the functional outcomes according to the different treatment options available.

\section{Conclusion}

Patients with glottis carcinoma and advanced oncological disease of the larynx and hypopharynx can be more prone to suffer hemilarynx fixation and require a pre-treatment tracheostomy. Therefore, and according to previous reports, these patients are poor candidates for organ preservation treatments. Moreover, persistent tracheostomy dependence after primary chemoradiation increases significantly the morbidity, and decreases the quality of life of those patients. Patients with glottis cancer are more prone to need a tracheostomy, but no statistical difference according to the 
oncological stage and the need for tracheostomy were detected. A more thorough patient selection is needed to improve the quality of life of our patients and reduce the permanent tracheostomy dependence.

\section{Conflict of Interest}

The authors have no conflicts of interest to declare.

\section{References}

1 Cattaruzza MS, Maisonneuve P, Boyle P. Epidemiology of laryngeal cancer. Eur J Cancer B Oral Oncol 1996;32B(05): 293-305

2 Cooper JS, Porter K, Mallin K, et al. National Cancer Database report on cancer of the head and neck: 10-year update. Head Neck 2009;31(06):748-758

3 Hoffman HT, Porter K, Karnell LH, et al. Laryngeal cancer in the United States: changes in demographics, patterns of care, and survival. Laryngoscope 2006;116(9 Pt 2, Suppl 111)1-13

4 Olsen KD. Reexamining the treatment of advanced laryngeal cancer. Head Neck 2010;32(01):1-7

5 Day D, Hansen AR, Siu LL. Hypopharyngeal cancer: looking back, moving forward. Curr Oncol 2016;23(04):221-222

6 Cmelak AJ, Li S, Goldwasser MA, et al. Phase II trial of chemoradiation for organ preservation in resectable stage III or IV squamous cell carcinomas of the larynx or oropharynx: results of Eastern Cooperative Oncology Group Study E2399. J Clin Oncol 2007;25(25):3971-3977

7 Guadagnolo BA, Haddad RI, Posner MR, et al. Organ preservation and treatment toxicity with induction chemotherapy followed by radiation therapy or chemoradiation for advanced laryngeal cancer. Am J Clin Oncol 2005;28(04):371-378

8 Woodson GE, Rosen CA, Murry T, et al. Assessing vocal function after chemoradiation for advanced laryngeal carcinoma. Arch Otolaryngol Head Neck Surg 1996;122(08):858-864
9 Lazarus CL, Logemann JA, Pauloski BR, et al. Swallowing disorders in head and neck cancer patients treated with radiotherapy and adjuvant chemotherapy. Laryngoscope 1996;106(9 Pt 1):1157-1166

10 Terrell JE, Ronis DL, Fowler KE, et al. Clinical predictors of quality of life in patients with head and neck cancer. Arch Otolaryngol Head Neck Surg 2004;130(04):401-408

11 Pfister DG, Spencer S, Brizel DM, et al; National Comprehensive Cancer Network. Head and neck cancers, Version 2.2014. Clinical practice guidelines in oncology. J Natl Compr Canc Netw 2014; 12(10):1454-1487

12 Forastiere AA, Goepfert H, Maor M, et al. Concurrent chemotherapy and radiotherapy for organ preservation in advanced laryngeal cancer. N Engl J Med 2003;349(22):2091-2098

13 Tennant PA, Cash E, Bumpous JM, Potts KL. Persistent tracheostomy after primary chemoradiation for advanced laryngeal or hypopharyngeal cancer. Head Neck 2014;36(11):1628-1633

14 The Department of Veterans Affairs Laryngeal Cancer Study Group. Induction chemotherapy plus radiation compared with surgery plus radiation in patients with advanced laryngeal cancer. N Engl J Med 1991;324(24):1685-1690

15 Weber RS, Berkey BA, Forastiere A, et al. Outcome of salvage total laryngectomy following organ preservation therapy: the Radiation Therapy Oncology Group trial 91-11. Arch Otolaryngol Head Neck Surg 2003;129(01):44-49

16 MacKenzie R, Franssen E, Balogh J, Birt D, Gilbert R. The prognostic significance of tracheostomy in carcinoma of the larynx treated with radiotherapy and surgery for salvage. Int J Radiat Oncol Biol Phys 1998;41(01):43-51

17 McNeil BJ, Weichselbaum R, Pauker SG. Speech and survival: tradeoffs between quality and quantity of life in laryngeal cancer. N Engl J Med 1981;305(17):982-987

18 Staton J, Robbins KT, Newman L, Samant S, Sebelik M, Vieira F. Factors predictive of poor functional outcome after chemoradiation for advanced laryngeal cancer. Otolaryngol Head Neck Surg 2002;127(01):43-47

19 Hutcheson KA, Alvarez CP, Barringer DA, Kupferman ME, Lapine PR, Lewin JS. Outcomes of elective total laryngectomy for laryngopharyngeal dysfunction in disease-free head and neck cancer survivors. Otolaryngol Head Neck Surg 2012;146(04):585-590 